\title{
Orlicz Generalized Difference Sequence Space and the Linked Pre-Quasi Operator Ideal
}

\author{
Awad A. Bakery $\mathbb{D}^{1,2}$ and OM Kalthum S. K. Mohamed $\mathbb{D}^{1,3}$ \\ ${ }^{1}$ University of Jeddah, College of Science and Arts at Khulis, Department of Mathematics, Jeddah, Saudi Arabia \\ ${ }^{2}$ Department of Mathematics, Faculty of Science, Ain Shams University, P. O. Box 1156, Cairo, 11566, Abbassia, Egypt \\ ${ }^{3}$ Academy of Engineering and Medical Sciences, Department of Mathematics, Khartoum, Sudan
}

Correspondence should be addressed to OM Kalthum S. K. Mohamed; om_kalsoom2020@yahoo.com

Received 10 November 2020; Revised 8 December 2020; Accepted 10 December 2020; Published 31 December 2020

Academic Editor: Dimitri Mugnai

Copyright ( 2020 Awad A. Bakery and OM Kalthum S. K. Mohamed. This is an open access article distributed under the Creative Commons Attribution License, which permits unrestricted use, distribution, and reproduction in any medium, provided the original work is properly cited.

\begin{abstract}
In this article, the necessary conditions on $s$-type Orlicz generalized difference sequence space to generate an operator ideal have been examined. Therefore, the $s$-type Orlicz generalized difference sequence space which fails to generate an operator ideal has been shown. We investigate the sufficient conditions on this sequence space to be premodular Banach special space of sequences, and the constructed pre-quasi operator ideal becomes small, simple, closed, Banach space and has eigenvalues identical with its $s$ numbers.
\end{abstract}

\section{Introduction}

The operator ideals have a wide field of mathematics in functional analysis, for instance, in eigenvalue distribution theorem, geometric structure of Banach spaces, and theory of fixed point. By $\mathbb{C}^{\mathbb{N}}, c, \ell_{\infty}, \ell_{r}$, and $c_{0}$, we denote the spaces of all, convergent, bounded, $r$-absolutely summable and null sequences of complex numbers, respectively. $\mathbb{N}$ indicates the set of nonnegative integers. Tripathy et al. [1] introduced and studied the forward and backward generalized difference sequence spaces:

$$
\begin{aligned}
U\left(\Delta_{n}^{(m)}\right) & =\left\{\left(w_{k}\right) \in \mathbb{C}^{\mathbb{N}}:\left(\Delta_{n}^{(m)} w_{k}\right) \in U\right\}, \\
U\left(\Delta_{n}^{m}\right) & =\left\{\left(w_{k}\right) \in \mathbb{C}^{\mathbb{N}}:\left(\Delta_{n}^{m} w_{k}\right) \in U\right\},
\end{aligned}
$$

where $m, n \in \mathbb{N}, U=\ell_{\infty}, c$ or $c_{0}$, with

$$
\begin{aligned}
\Delta_{n}^{(m)} w_{k} & =\sum_{\nu=0}^{m}(-1)^{\nu} C_{\nu}^{m} w_{k+\nu n}, \\
\Delta_{n}^{m} w_{k} & =\sum_{\nu=0}^{m}(-1)^{\nu} C_{\nu}^{m} w_{k-\nu n},
\end{aligned}
$$

respectively. When $n=1$, the generalized difference sequence spaces reduced to $U\left(\Delta^{(m)}\right)$ defined and investigated by Et and Çolak [2]. If $m=1$, the generalized difference sequence spaces reduced to $U\left(\Delta_{n}\right)$ defined and investigated by Tripathy and Esi [3]. While if $n=1$ and $m=1$, the generalized difference sequence spaces reduced to $U(\Delta)$ defined and studied by Kizmaz [4].

Definition 1 (see [5]). The backward generalized difference $\Delta_{n+1}^{m}$ is called an absolute nondecreasing; if $\left|x_{i}\right| \leq\left|y_{i}\right|$ for all $i \in \mathbb{N}$, then $\left|\Delta_{n+1}^{m}\right| x_{i}|| \leq\left|\Delta_{n+1}^{m}\right| y_{i}||$.

An Orlicz function [6] is a function $\psi:[0, \infty) \longrightarrow[0, \infty)$, which is convex, continuous, and nondecreasing with $\psi(0)=0, \psi(u)>0$ for $u>0$ and $\psi(u) \longrightarrow \infty$, as $u \longrightarrow \infty$. An Orlicz function $\psi$ [7] is said to satisfy $\delta_{2}$-condition for all values of $x \geq 0$, if there exists a constant $k>0$, such that $\psi(2 x) \leq k \psi(x)$. The $\delta_{2}$-condition is equivalent to $\psi(l x) \leq k l \psi(x)$, for all values of $x$ and for $l>1$. Lindentrauss and Tzafriri [8] utilized the idea of an Orlicz function to define Orlicz sequence space: 


$$
\ell_{\psi}=\left\{u \in \mathbb{C}^{\mathbb{N}}: \rho(\beta u)<\infty, \quad \text { for some } \beta>0\right\}, \quad \text { where } \rho(u)=\sum_{k=0}^{\infty} \psi\left(\left|u_{k}\right|\right)
$$

where $\left(\ell_{\psi},\|\|.\right)$ is a Banach space with the Luxemburg norm:

$$
\|u\|=\inf \left\{\beta>0: \rho\left(\frac{u}{\beta}\right) \leq 1\right\} \text {. }
$$

Every Orlicz sequence space contains a subspace that is isomorphic to $c_{0}$ or $\ell^{q}$, for some $1 \leq q<\infty$.

Let $r=\left(r_{j}\right) \in \mathbb{R}^{+\mathbb{N}}$, where $\mathbb{R}^{+\mathbb{N}}$ indicates the space of sequences with positive real numbers, and we define the Orlicz backward generalized difference sequence space as follows:

$$
\left(\ell_{\psi}\left(\Delta_{n+1}^{m}\right)\right)_{\tau}=\left\{w=\left(w_{j}\right) \in \mathbb{C}^{\mathbb{N}}: \exists \sigma>0, \quad \text { with } \tau(\sigma w)<\infty\right\},
$$

where $\quad \tau(w)=\sum_{j=0}^{\infty} \psi\left(\left|\Delta_{n+1}^{m}\right| w_{j}||\right), \quad w_{j}=0 \quad$ for $\quad j<0$, $\Delta_{n+1}^{m}\left|w_{j}\right|=\Delta_{n+1}^{m-1}\left|w_{j}\right|-\Delta_{n+1}^{m-1}\left|w_{j-1}\right|$, and $\Delta^{0} w_{j}=w_{j}$, for all $j, n, m \in \mathbb{N}$. It is a Banach space, with

$$
\|w\|=\inf \left\{\sigma>0: \tau\left(\frac{w}{\sigma}\right) \leq 1\right\} .
$$

When $\psi(w)=w^{r}$, then $\ell_{\psi}\left(\Delta_{n+1}^{m}\right)=\ell_{r}\left(\Delta_{n+1}^{m}\right)$ studied in [5]. Mohiuddine et al. [9] investigated the applications of fractional-order difference operators by constructing Orlicz almost null and almost convergent sequence spaces. Yaying et al. [10] examined sequence spaces generated by the triple band generalized Fibonacci difference operator. By $\mathscr{B}(W, Z)$, we will indicate the set of every operators which are linear and bounded between Banach spaces $W$ and $Z$, and if $W=Z$, we write $\mathscr{B}(W)$. The $s$-numbers [11] have many examples such as the $r$-th approximation number, denoted by $\alpha_{r}(V)$, which is defined by $\alpha_{r}(V)=\inf \{\|V-B\|: B \in \mathscr{B}(W, Z)$ and $\operatorname{rank}(B) \leq r\}, \quad$ and the $r$-th Kolmogorov number, denoted by $d_{r}(V)$, which is defined by $d_{r}(V)=\inf _{\operatorname{dim} W \leq r} \sup _{\|w\| \leq 1} \inf _{v \in W}\|V w-v\|$. The following notations will be used in the sequel:

$$
\begin{aligned}
& X^{S}:=\left\{X^{S}(W, Z)\right\}, \quad \text { where } X^{S}(W, Z):=\left\{V \in \mathscr{B}(W, Z):\left(\left(s_{j}(V)\right)_{j=0}^{\infty} \in X\right\},\right. \\
& X^{\text {app }}:=\left\{X^{\text {app }}(W, Z)\right\}, \quad \text { where } X^{\text {app }}(W, Z):=\left\{V \in \mathscr{B}(W, Z):\left(\left(\alpha_{j}(V)\right)_{j=0}^{\infty} \in X\right\},\right. \\
& X^{\mathrm{Kol}}:=\left\{X^{\mathrm{Kol}}(W, Z)\right\}, \quad \text { where } X^{\mathrm{Kol}}(W, Z):=\left\{V \in \mathscr{B}(W, Z):\left(\left(d_{j}(V)\right)_{j=0}^{\infty} \in X\right\},\right. \\
& X^{v}:=\left\{X^{v}(W, Z)\right\}, \quad \text { where } X^{v}(W, Z):=\left\{\begin{array}{l}
V \in \mathscr{B}(W, Z):\left(\left(v_{j}(V)\right)_{j=0}^{\infty} \in X,\right. \\
\left\|V-v_{j}(V) I\right\|=0, \quad \text { for all } j \in \mathbb{N}
\end{array}\right\} .
\end{aligned}
$$

A few of operator ideals in the class of Hilbert spaces or Banach spaces are defined by distinct scalar sequence spaces, such as the ideal of compact operators $\mathscr{B}_{c}$ formed by $\left(d_{r}(V)\right)$ and $c_{0}$. Pietsch [11] studied the quasi-ideals $\left(\ell_{r}\right)^{\text {app }}$ for $r \in(0, \infty)$, the ideals of Hilbert Schmidt operators between Hilbert spaces constructed by $\ell_{2}$, and the ideals of nuclear operators generated by $\ell_{1}$. He explained that $\bar{\delta}=$ $\left(\ell_{r}\right)^{\text {app }}$ for $r \in[1, \infty)$, where $\overline{\mathcal{S}}$ is the closed class of all finite rank operators, and the class $\left(\ell_{r}\right)^{\text {app }}$ became simple Banach and small [12]. The strictly inclusion $\left(\ell_{r}\right)^{\text {app }}(W, Z) \subsetneq$ $\left(\ell_{j}\right)^{\text {app }}(W, Z) \mathscr{B}(W, Z)$, whenever $j>r>0, W$ and $Z$ are infinite dimensional Banach spaces investigated through Makarov and Faried [13]. Faried and Bakery [14] gave a generalization of the class of quasi-operator ideal which is the pre-quasi operator ideal, and they examined several geometric and topological structures of $\left(\ell_{M}\right)^{\mathrm{S}}$ and $(\operatorname{ces}(r))^{\mathrm{S}}$. Başarir and Kara [15] studied the compact operators on some Euler $B(m)$-difference sequence spaces. İlkhan et al. [16] investigated the multiplication operators on Cesáro second-order function spaces. The point of this article to explain some results of $\left(\ell_{\psi}\left(\Delta_{n+1}^{m}\right)\right)_{\tau}$ equipped with a prequasi norm $\tau$. Firstly, we give the necessary conditions on any $s$-type $\left(\ell_{\psi}\left(\Delta_{n+1}^{m}\right)\right)_{\tau}$ to give an operator ideal. Secondly, some geometric and topological structures of $\left(\ell_{\psi}\left(\Delta_{n+1}^{m}\right)\right)_{\tau}^{\mathrm{S}}$ have been studied, such as closed, small, simple Banach and $\left(\ell_{\psi}\left(\Delta_{n+1}^{m}\right)\right)^{S}=\left(\ell_{\psi}\left(\Delta_{n+1}^{m}\right)\right)^{\nu}$. A strictly inclusion relation of $\left(\ell_{\psi}\left(\Delta_{n+1}^{m}\right)\right)^{S}$ has been determined for different Orlicz functions and $\Delta_{n+1}^{m}$.

\section{Preliminaries and Definitions}

Definition 2 (see [11]). An operator $V \in \mathscr{B}(W)$ is called approximable if there are $D_{r} \in \mathcal{S}(W)$, for every $r \in \mathbb{N}$ and $\lim _{r \rightarrow \infty}\left\|V-D_{r}\right\|=0$.

By $\Upsilon(W, Z)$, we will indicate the space of all approximable operators from $W$ to $Z$. The sequence $e_{j}=(0,0, \ldots, 1,0,0, \ldots)$ with 1 in the $j$-th coordinate, for every $j \in \mathbb{N}$, will be used in the sequel.

Lemma 1 (see [11]). Let $V \in \mathscr{B}(W, Z)$. If $V \notin \Upsilon(W, Z)$, then there are $G \in \mathscr{B}(W)$ and $B \in \mathscr{B}(Z)$ such that $B V G e_{r}=e_{r}$, for all $r \in \mathbb{N}$.

Definition 3 (see [11]). A Banach space $W$ is called simple if $\mathscr{B}(W)$ includes one and only one nontrivial closed ideal. 
Theorem 1 (see [11]). If $W$ is Banach space with $\operatorname{dim}(W)=\infty$, then

$$
\mathcal{S}(W) \subsetneq \Upsilon(W) \subsetneq \mathscr{B}_{c}(W) \mathscr{B}(W) .
$$

Definition 4 (see[14]). The space of linear sequence spaces $\mathbb{Y}$ is called a special space of sequences (sss) if

(1) $e_{r} \in \mathbb{Y}$ with $r \in \mathbb{N}$,

(2) let $u=\left(u_{r}\right) \in \mathbb{C}^{\mathbb{N}}, v=\left(v_{r}\right) \in \mathbb{Y}$, and $\left|u_{r}\right| \leq\left|v_{r}\right|$, for every $r \in \mathbb{N}$, then $u \in \mathbb{Y}$. This means $\mathbb{Y}$ be solid, and

(3) if $\left(u_{r}\right)_{r=0}^{\infty} \in \mathbb{Y}$, then $\left(u_{[r / 2]}\right)_{r=0}^{\infty} \in \mathbb{Y}$, wherever $[r / 2]$ means the integral part of $r / 2$.

Definition 5 (see [5]). A subspace of the (sss) $\Psi_{\tau}$ is called a premodular (sss) if there is a function $\tau: \mathbb{Y} \longrightarrow[0, \infty)$ verifying the following conditions:

(i) $\tau(y) \geq 0$ for each $y \in \mathbb{Y}$ and $\tau(y)=0 \Leftrightarrow y=\theta$, where $\theta$ is the zero element of $\mathbb{Y}$

(ii) There exists $a \geq 1$ such that $\tau(\eta y) \leq a|\eta| \tau(y)$, for all $y \in \mathbb{Y}$ and $\eta \in \mathbb{C}$

(iii) For some $b \geq 1, \tau(y+z) \leq b(\tau(y)+\tau(z))$, for every $y, z \in \mathbb{Y}$

(iv) $\left|y_{r}\right| \leq\left|z_{r}\right|$ with $r \in \mathbb{N}$, which implies that $\tau\left(\left(y_{r}\right)\right) \leq \tau\left(\left(z_{r}\right)\right)$

(v) For some $b_{0} \geq 1, \tau\left(\left(y_{r}\right)\right) \leq \tau\left(\left(y_{[r / 2]}\right)\right) \leq b_{0} \tau\left(\left(y_{i}\right)\right)$

(vi) If $y=\left(y_{r}\right)_{r=o}^{\infty} \in \mathbb{Y}$ and $d>0$, then there is $r_{0} \in \mathbb{N}$ with $\tau\left(\left(y_{r}\right)_{r=r_{0}}^{\infty}\right)<d$

(vii) There is $t>0$ with $\tau(\nu, 0,0,0, \ldots) \geq t|\nu| \tau(1,0,0,0$, $\ldots)$, for any $v \in \mathbb{C}$

The (sss) $\bigvee_{\tau}$ is called pre-quasi normed (sss) if $\tau$ satisfies Parts (i)-(iii) of Definition 5 and when the space $\mathbb{Y}$ is complete under $\tau$, then $\mathbb{Y}_{\tau}$ is called a pre-quasi Banach (sss).

Theorem 2 (see [5]). A pre-quasi norm (sss) $\mathbb{Y}_{\tau}$, whenever it is premodular (sss).

By $\mathscr{B}$, we will denote the class of all bounded linear operators between any pair of Banach spaces.

Definition 6 (see [5]). A class $\mathscr{R} \subseteq \mathscr{B}$ is called an operator ideal if every $\mathscr{R}(W, Z)=\mathscr{R} \cap \mathscr{B}(W, Z)$ satisfies the following conditions:

(i) $\mathscr{R} \supseteq \mathcal{S}$

(ii) The space $\mathscr{R}(W, Z)$ is linear over $\mathbb{C}$

(iii) If $V \in \mathscr{B}\left(W_{0}, W\right), G \in \mathscr{R}(W, Z)$, and $Q \in \mathscr{B}(Z$, $\left.Z_{0}\right)$, then $Q G V \in \mathscr{R}\left(W_{0}, Z_{0}\right)$, where $W_{0}$ and $Z_{0}$ are Banach spaces
Definition 7 (see [5]). A pre-quasi norm on the ideal $B$ is a function $\zeta: B \longrightarrow[0, \infty)$ which satisfies the following conditions:

(1) For all $V \in B(W, Z), \zeta(V) \geq 0$ and $\zeta(V)=0$ if and only if $V=0$

(2) There is $H \geq 1$ such that $\zeta(\eta V) \leq H|\eta| \zeta(V)$, for all $V \in B(W, Z)$ and $\eta \in \mathbb{C}$

(3) There is $b \geq 1$ such that $\zeta\left(V_{1}+V_{2}\right) \leq$ $b\left[\zeta\left(V_{1}\right)+\zeta\left(V_{2}\right)\right]$, for all $V_{1}, V_{2} \in B(W, Z)$

(4) There is $D \geq 1$ such that if $U \in \mathscr{B}\left(W_{0}, W\right)$, $T \in B(W, Z)$, and $V \in \mathscr{B}\left(Z, Z_{0}\right)$, then $\zeta(\mathrm{VTU}) \leq$ $D\|V\| \zeta(T)\|U\|$

Theorem 3 (see [14]). The class $X_{\tau}^{\mathrm{S}}$ is an operator ideal, if $X_{\tau}$ is a (sss).

Theorem 4 (see [14]). The function $\zeta(V)=\tau\left(s_{r}(V)\right)_{r=0}^{\infty}$ forms a pre-quasi norm on $X_{\tau}^{S}$, whenever $X_{\tau}$ be a premodular (sss).

The inequality [17] $\left|a_{i}+b_{i}\right|^{q_{i}} \leq H\left(\left|a_{i}\right|^{q_{i}}+\left|b_{i}\right|^{q_{i}}\right)$, where $q_{i} \geq 0$ for all $i \in \mathbb{N}, H=\max \left\{1,2^{h-1}\right\}$ and $h=\sup _{i} q_{i}$, will be used in the sequel.

\section{Main Results}

We give the necessary conditions on $s$-type $\ell_{\psi}\left(\Delta_{n+1}^{m}\right)$ under $\tau: \ell_{\psi}\left(\Delta_{n+1}^{m}\right) \longrightarrow[0, \infty)$ such that $\left(\ell_{\psi}\left(\Delta_{n+1}^{m}\right)\right)_{\tau}^{S^{n+1}}$ forms an operator ideal.

Theorem 5. For $\left(\ell_{\psi}\left(\Delta_{n+1}^{m}\right)\right)_{\tau}=\left\{x=\left(s_{n}(V)\right) \in \mathbb{C}^{\mathbb{N}}: V \in \mathscr{B}\right.$ $(W, Z)$ and $\tau(x)<\infty\}$. If $\left(\ell_{\psi}\left(\Delta_{n+1}^{m}\right)\right)_{\tau}^{\mathrm{S}}$ is an operator ideal, then the following conditions are satisfied:

(1) The set $\left(\ell_{\psi}\left(\Delta_{n+1}^{m}\right)\right)_{\tau}$ contains $F$, the space of all the sequences with finite nonzero numbers

(2) If $\left(s_{r}\left(V_{1}\right)\right)_{r=0}^{\infty} \in\left(\ell_{\psi}\left(\Delta_{n+1}^{m}\right)\right)_{\tau}$ and $\left(s_{r}\left(V_{2}\right)\right)_{r=0}^{\infty} \in$ $\left(\ell_{\psi}\left(\Delta_{n+1}^{m}\right)\right)_{\tau}$, then $\left(s_{r}\left(V_{1}+V_{2}\right)\right)_{r=0}^{\infty} \in\left(\ell_{\psi}\left(\Delta_{n+1}^{m}\right)\right)_{\tau}$

(3) For all $\lambda \in \mathbb{C}$ and $\left(s_{r}(V)\right)_{r=0}^{\infty} \in\left(\ell_{\psi}\left(\Delta_{n+1}^{m}\right)\right)_{\tau}$, then $|\lambda|\left(s_{r}(V)\right)_{r=0}^{\infty} \in\left(\ell_{\psi}\left(\Delta_{n+1}^{m}\right)\right)_{\tau}$

(4) The sequence space $\left(\ell_{\psi}\left(\Delta_{n+1}^{m}\right)\right)_{\tau}$ is solid

Proof. let $\left(\ell_{\psi}\left(\Delta_{n+1}^{m}\right)\right)_{\tau}^{\mathrm{S}}$ be an operator ideal.

(i) We have $\mathcal{S}(W, Z) \subset\left(\ell_{\psi}\left(\Delta_{n+1}^{m}\right)\right)^{\mathrm{S}}(W, Z)$. Hence, for all $T \in \mathcal{S}(W, Z)$, we have $\left(s_{r}(V)\right)_{r=0}^{\infty} \in F$. This gives that $\left(s_{r}(V)\right)_{r=0}^{\infty} \in\left(\ell_{\psi}\left(\Delta_{n+1}^{m}\right)\right)_{\tau}$. Hence, $F \subset\left(\ell_{\psi}\left(\Delta_{n+1}^{m}\right)\right)_{\tau}$.

(ii) The space $\left(\ell_{\psi}\left(\Delta_{n+1}^{m}\right)\right)_{\tau}^{\mathrm{S}}(W, Z)$ is linear over $\mathbb{C}$. Hence, for each $\lambda \in \mathbb{C}^{\tau}$ and $V_{1}, V_{2} \in\left(\ell_{\psi}\left(\Delta_{n+1}^{m}\right)\right)_{\tau}^{\mathrm{S}}$ $(W, Z)$, we have $V_{1}+V_{2} \in\left(\ell_{\psi}\left(\Delta_{n+1}^{m}\right)\right)^{{ }^{\prime}}(W$, $Z)$ and $\lambda V_{1} \in\left(\ell_{\psi}\left(\Delta_{n+1}^{m}\right)\right)_{\tau}^{S}(W, Z)$. This implies that 


$$
\begin{aligned}
\left(s_{r}\left(V_{1}\right)\right)_{r=0}^{\infty} & \in\left(\ell_{\psi}\left(\Delta_{n+1}^{m}\right)\right)_{\tau}, \\
\left(s_{r}\left(V_{2}\right)\right)_{r=0}^{\infty} & \in\left(\ell_{\psi}\left(\Delta_{n+1}^{m}\right)\right)_{\tau} \Longrightarrow\left(s_{r}\left(V_{1}+V_{2}\right)\right)_{r=0}^{\infty} \in\left(\ell_{\psi}\left(\Delta_{n+1}^{m}\right)\right)_{\tau^{\prime}} \\
\lambda & \in \mathbb{C}, \\
\left(s_{r}\left(V_{1}\right)\right)_{r=0}^{\infty} & \in\left(\ell_{\psi}\left(\Delta_{n+1}^{m}\right)\right)_{\tau} \Longrightarrow|\lambda|\left(s_{r}\left(V_{1}\right)\right)_{r=0}^{\infty} \in\left(\ell_{\psi}\left(\Delta_{n+1}^{m}\right)\right)_{\tau} .
\end{aligned}
$$

(iii) If $A \in \mathscr{B}\left(W_{0}, W\right), B \in\left(\ell_{\psi}\left(\Delta_{n+1}^{m}\right)\right)_{\tau}^{\mathrm{S}}(W, Z)$, and $D \in \mathscr{B}\left(Z, Z_{0}\right)$, then DBA $\in\left(\ell_{\psi}\left(\Delta_{n+1}^{m^{\tau}}\right)\right)_{\tau}^{\mathrm{S}}\left(W_{0}, Z_{0}\right)$, where $W_{0}$ and $Z_{0}$ are arbitrary Banach spaces. Therefore, if $A \in \mathscr{B}\left(W_{0}, W\right)$, $\left(s_{r}(B)\right)_{r=0}^{\infty} \in\left(\ell_{\psi}\left(\Delta_{n+1}^{m}\right)\right)_{\tau}$, and $D \in \mathscr{B}\left(Z, Z_{0}\right)$, then $\left(s_{r}(\mathrm{DBA})\right)_{r=0}^{\infty} \in\left(\ell_{\psi}\left(\Delta_{n+1}^{m}\right)\right)_{\tau}$. In addition, $s_{r}(\mathrm{DBA}) \leq\|D\| s_{r}(B)\|A\|$. By using condition 3, if $\left(\|D\|\|A\| s_{r}(B)\right)_{r=0}^{\infty} \in\left(\ell_{\psi}\left(\Delta_{n+1}^{m}\right)\right)_{\tau}$, we have $\left(s_{r}(\mathrm{DBA})\right)_{r=0}^{\infty} \in\left(\ell_{\psi}\left(\Delta_{n+1}^{m}\right)\right)_{\tau}$. This means that $\left(\ell_{\psi}\left(\Delta_{n+1}^{m}\right)\right)_{\tau}$ is solid.

We explain that for any backward generalized difference $\Delta_{n+1}^{m}$, the space $\left(\ell_{\psi}\left(\Delta_{n+1}^{m}\right)\right)_{\tau}^{S}$ is not operator ideal.

Theorem 6. The space $\left(\ell_{\psi}\left(\Delta_{n+1}^{m}\right)\right)_{\tau}^{S}$ is not operator ideal, where $\psi$ is an Orlicz function satisfying $\delta_{2}$-condition and $\tau(w)=\sum_{j=0}^{\infty} \psi\left(\left|\Delta_{n+1}^{m}\right| w_{j}||\right)$, for all $w \in \ell_{\psi}\left(\Delta_{n+1}^{m}\right)$.
Proof. if we choose $m=2, n=1, w_{k}=1$, and $v_{k}=w_{k}$ for $k=3 s$ or $v_{k}=0$, otherwise, for all $s, k \in \mathbb{N}$. We have $\left|v_{k}\right| \leq\left|w_{k}\right|$, for all $k \in \mathbb{N}, w \in\left(\ell_{\psi}\left(\Delta_{2}^{2}\right)\right)_{\tau}$, and $v \notin\left(\ell_{\psi}\left(\Delta_{2}^{2}\right)\right)_{\tau}$. Hence, the space $\left(\ell_{\psi}\left(\Delta_{n+1}^{m}\right)\right)_{\tau}$ is not solid. This finishes the proof.In this part, we give the conditions on Orlicz backward generalized difference sequence space to be premodular Banach (sss).

Theorem 7. If $\psi$ is an Orlicz function satisfying $\delta_{2}$-condition and $\Delta_{n+1}^{m}$ is an absolute nondecreasing, then the space $\left(\ell_{\psi}\left(\Delta_{n+1}^{m}\right)\right)_{\tau}$ is a premodular Banach (sss), where

$$
\tau(w)=\sum_{j=0}^{\infty} \psi\left(\left|\Delta_{n+1}^{m}\right| w_{j}||\right), \quad \text { for all } w \in \ell_{\psi}\left(\Delta_{n+1}^{m}\right) .
$$

Proof. (1)

(i) Suppose $v, w \in \ell_{\psi}\left(\Delta_{n+1}^{m}\right)$. Since $\psi$ is nondecreasing, convex, and satisfying $\delta_{2}$-condition and $\Delta_{n+1}^{m}$ is an absolute nondecreasing, then there exists a number $b>0$ such that

$$
\begin{aligned}
\tau(v+w) & =\sum_{i=0}^{\infty} \psi\left(\left|\Delta_{n+1}^{m}\right| v_{i}+w_{i}||\right) \leq \sum_{i=0}^{\infty} \psi\left(\left|\Delta_{n+1}^{m}\right| v_{i}||+\left|\Delta_{n+1}^{m}\right| w_{i}||\right) \\
& \leq \frac{1}{2}\left(\sum_{i=0}^{\infty} \psi\left(2\left|\Delta_{n+1}^{m}\right| v_{i}||\right)+\sum_{i=0}^{\infty} \psi\left(2\left|\Delta_{n+1}^{m}\right| w_{i}||\right) \leq \frac{b}{2}(\tau(v)+\tau(w)) \leq B(\tau(v)+\tau(w))<\infty\right.
\end{aligned}
$$

for some $B=\max \{1, b / 2\}$. Then, $v+w \in \ell_{\psi}\left(\Delta_{n+1}^{m}\right)$.

(ii) Assume $\lambda \in \mathbb{C}$ and $v \in \ell_{\psi}\left(\Delta_{n+1}^{m}\right)$. Since $\psi$ is satisfying $\delta_{2}$-condition, we have

$$
\tau(\lambda v)=\sum_{i=0}^{\infty} \psi\left(\left|\Delta_{n+1}^{m}\right| \lambda v_{r}||\right) \leq d|\lambda| \sum_{i=0}^{\infty} \psi\left(\left|\Delta_{n+1}^{m}\right| v_{r}||\right) \leq D|\lambda| \tau(v)<\infty
$$

where $D=\max \{1, d\}$. Then, $\lambda v \in \ell_{\psi}\left(\Delta_{n+1}^{m}\right)$. Hence, from Parts (i) and (iii), the space $\ell_{\psi}\left(\Delta_{n+1}^{m}\right)$ is linear. Therefore, $e_{r} \in \ell_{q} \subseteq \ell_{\psi} \subseteq \ell_{\psi}\left(\Delta_{n+1}^{m}\right)$, for all $r \in \mathbb{N}$ and $q \geq 1$. Therefore, $e_{r} \in \ell_{\psi}\left(\Delta_{n+1}^{m}\right)$ for all $r \in \mathbb{N}$.
(2) Suppose $\left|x_{i}\right| \leq\left|y_{i}\right|$, for all $i \in \mathbb{N}$ and $y \in \ell_{\psi}\left(\Delta_{n+1}^{m}\right)$. $\psi$ is nondecreasing, and $\Delta_{n+1}^{m}$ is an absolute nondecreasing. Hence, we have

$$
\tau(x)=\sum_{i=0}^{\infty} \psi\left(\left|\Delta_{n+1}^{m}\right| x_{i}||\right) \leq \sum_{i=0}^{\infty} \psi\left(\left|\Delta_{n+1}^{m}\right| y_{i}||\right)=\tau(y)<\infty,
$$


so $x \in \ell_{\psi}\left(\Delta_{n+1}^{m}\right)$.

(3) Assume that $\left(v_{r}\right) \in \ell_{\psi}\left(\Delta_{n+1}^{m}\right)$. We have

$$
\tau\left(\left(v_{[}\left[\frac{r}{2}\right]\right)\right)=\sum_{i=0}^{\infty} \psi\left(\left|\Delta_{n+1}^{m}\right| v_{\left[\frac{r}{2}\right]}||\right) \leq 2 \sum_{i=0}^{\infty} \psi\left(\left|\Delta_{n+1}^{m}\right| v_{r}||\right)=2 \tau(v)
$$

then $\left(v_{[r / 2]}\right) \in \ell_{\psi}\left(\Delta_{n+1}^{m}\right)$.

(i) Obviously, $\tau(w) \geq 0$ and $\tau(w)=0 \Leftrightarrow w=\theta$

(ii) There is $D \geq 1$ where $\tau(\eta w) \leq D|\eta| \tau(w)$, for all $w \in \ell_{\psi}\left(\Delta_{n+1}^{m}\right)$ and $\eta \in \mathbb{C}$

(iii) For some $B \geq 1$, we have $\tau(v+w) \leq B(\tau(v)+\tau(w))$, for all $v, w \in \ell_{\psi}\left(\Delta_{n+1}^{m}\right)$

(iv) Clearly from the proof part (2)

(v) From (3), we have that $b_{0}=2 \geq 1$

(vi) It is obvious that $\bar{F}=\ell_{\psi}\left(\Delta_{n+1}^{m}\right)$

(vii) Since $\psi$ is satisfying $\delta_{2}$-condition, there is $\zeta$ with $0<\zeta \leq(\psi(|\eta|) / \eta)$ such that $\tau(\eta, 0,0,0, \ldots) \geq \zeta|\eta| \tau(1,0,0,0, \ldots), \quad$ for each $\eta \neq 0$ and $\zeta>0$, if $\eta=0$

Hence, the space $\left(\ell_{\psi}\left(\Delta_{n+1}^{m}\right)\right)_{\tau}$ is premodular (sss). To explain that $\left(\ell_{\psi}\left(\Delta_{n+1}^{m}\right)\right)_{\tau}$ is a premodular Banach (sss), assume $x^{i}=\left(x_{k}^{i}\right)_{k=0}^{\infty}$ is a Cauchy sequence in $\left(\ell_{\psi}\left(\Delta_{n+1}^{m}\right)\right)_{\tau}$, and then for each $\varepsilon \in(0,1)$, there is $i_{0} \in \mathbb{N}$ such that for all $i, j \geq i_{0}$, we have

$$
\tau\left(x^{i}-x^{j}\right)=\sum_{k=0}^{\infty} \psi\left(\left|\Delta_{n+1}^{m}\right| x_{k}^{i}-x_{k}^{j}||\right)<\psi(\varepsilon) .
$$

Since $\psi$ is nondecreasing, hence, for $i, j \geq i_{0}$ and $k \in \mathbb{N}$, we conclude

$$
\left|\Delta_{n+1}^{m}\right| x_{k}^{i}\left|-\Delta_{n+1}^{m}\right| x_{k}^{j}||<\varepsilon .
$$

Therefore, $\left(\Delta_{n+1}^{m}\left|x_{k}^{j}\right|\right)$ is a Cauchy sequence in $\mathbb{C}$ for fixed $k \in \mathbb{N}$, so $\lim _{j \rightarrow \infty} \Delta_{n+1}^{m} x_{k}^{j}=\Delta_{n+1}^{m} x_{k}^{0}$ for fixed $k \in \mathbb{N}$. Hence, $\tau\left(x^{i}-x^{0}\right)<\psi(\varepsilon)$, for all $i \geq i_{0}$. Finally, to show that $x^{0} \in \ell_{\psi}\left(\Delta_{n+1}^{m}\right)$, we have

$$
\tau\left(x^{0}\right)=\tau\left(x^{0}-x^{n}+x^{n}\right) \leq B\left(\tau\left(x^{n}-x^{0}\right)+\tau\left(x^{n}\right)\right)<\infty .
$$

Therefore, $x^{0} \in \ell_{\psi}\left(\Delta_{n+1}^{m}\right)$. This gives that $\left(\ell_{\psi}\left(\Delta_{n+1}^{m}\right)\right)_{\tau}$ is a premodular Banach (sss).

In view of Theorem 2, we conclude the following theorem.

Theorem 8. If $\psi$ is an Orlicz function satisfying $\delta_{2}$-condition and $\Delta_{n+1}^{m}$ is an absolute nondecreasing, then the space $\left(\ell_{\psi}\left(\Delta_{n+1}^{m}\right)\right)_{\tau}$ is pre-quasi Banach (sss), where

$$
\tau(x)=\sum_{j=0}^{\infty} \psi\left(\left|\Delta_{n+1}^{m}\right| x_{j}||\right) \text {, for all } w \in \ell_{\psi}\left(\Delta_{n+1}^{m}\right) \text {., for all } x \in \ell_{\psi}\left(\Delta_{n+1}^{m}\right) .
$$

Corollary 1. If $0<p<\infty$ and $\Delta_{n+1}^{m}$ is an absolute nondecreasing, then $\left(\ell_{p}\left(\Delta_{n+1}^{m}\right)\right)_{\tau}$ is a premodular Banach (sss), where $\tau(x)=\sum_{i=0}^{\infty}\left|\Delta_{n+1}^{m}\right| x_{i}||^{p}$, for all $x \in \ell_{p}\left(\Delta_{n+1}^{m}\right)$.

\section{Pre-Quasi Banach Closed Ideal}

We introduce the sufficient conditions on $\ell_{\psi}\left(\Delta_{n+1}^{m}\right)$ such that the class $\left(\ell_{\psi}\left(\Delta_{n+1}^{m}\right)\right)_{\tau}^{\mathrm{S}}$ is Banach and closed.

Theorem 9. If $\psi$ is an Orlicz function satisfying $\delta_{2}$-condition and $\Delta_{n+1}^{m}$ is an absolute nondecreasing, then $\left(\left(\ell_{\psi}\left(\Delta_{n+1}^{m}\right)\right)_{\tau}^{\mathrm{S}}, \zeta\right)$ is a pre-quasi Banach operator ideal, with $\tau(w)=\sum_{j=0}^{\infty} \psi\left(\left|\Delta_{n+1}^{m}\right| w_{j}||\right), \quad$ for all $w \in \ell_{\psi}\left(\Delta_{n+1}^{m}\right)$ and $\zeta(V)=\tau\left(\left(s_{n}(V)\right)_{n=0}^{\infty}\right)$.

Proof. let the conditions be satisfied. Hence, from Theorems 3,4 , and 7 , the function $\zeta$ is a pre-quasi norm on the ideal $\left(\ell_{\psi}\left(\Delta_{n+1}^{m}\right)\right)^{S}$. Let $\left(V_{j}\right)$ be a Cauchy sequence in $\left(\ell_{\psi}\left(\Delta_{n+1}^{m}\right)\right)_{\tau}^{\bar{\xi}}(W, Z)$. Since $\mathscr{B}(W, Z) \supseteq\left(\ell_{\psi}\left(\Delta_{n+1}^{m}\right)\right)_{\tau}^{\mathrm{S}}(W, Z)$, we have

$$
\zeta\left(V_{i}-V_{j}\right)=\sum_{k=0}^{\infty} \psi\left(\left|\Delta_{n+1}^{m} s_{k}\left(V_{i}-V_{j}\right)\right|\right) \geq \psi\left(\left|\Delta_{n+1}^{m}\left(\left\|V_{i}-V_{j}\right\|\right)\right|\right) .
$$


Therefore, $\left(V_{j}\right)_{j \in \mathbb{N}}$ is a Cauchy sequence in $\mathscr{B}(W, Z)$. Since $\mathscr{B}(W, Z)$ is a Banach space, hence $T \in \mathscr{B}(W, Z)$ with $\lim _{j \longrightarrow \infty}\left\|V_{j}-V\right\|=0$ and while $\left(s_{n}\left(V_{i}\right)\right)_{n=0}^{\infty} \in\left(\ell_{\psi}\left(\Delta_{n+1}^{m}\right)\right)_{\tau}$, for each $i \in \mathbb{N}$. From Parts (ii), (iii), and (iv) of Definition 5, we have

$$
\begin{aligned}
\zeta(V) & =\sum_{r=0}^{\infty} \psi\left(\left|\Delta_{n+1}^{m} s_{r}\left(V-V_{j}+V_{j}\right)\right|\right) \leq B\left(\sum_{r=0}^{\infty} \psi\left(\left|\Delta_{n+1}^{m} s\left[\frac{r}{2}\right]\left(V-V_{j}\right)\right|\right)+\sum_{r=0}^{\infty} \psi\left(\left|\Delta_{n+1}^{m} s_{\left[\frac{r}{2}\right]}\left(V_{j}\right)\right|\right)\right) \\
& \leq B \sum_{r=0}^{\infty} \psi\left(\left|\Delta_{n+1}^{m} \| V-V_{j}\right| \mid\right)+B b_{0} \sum_{r=0}^{\infty} \psi\left(\left|\Delta_{n+1}^{m} s_{r}\left(V_{j}\right)\right|\right)<\varepsilon .
\end{aligned}
$$

\begin{abstract}
Therefore, $V \in\left(\ell_{\psi}\left(\Delta_{n+1}^{m}\right)\right)_{\tau}^{\mathrm{S}}(W, Z)$.

Theorem 10. If $\psi$ is an Orlicz function satisfying $\delta_{2}$-condition and $\Delta_{n+1}^{m}$ is an absolute nondecreasing, then $\left(\left(\ell_{\psi}\left(\Delta_{n+1}^{m}\right)\right)_{\tau}^{S}, \zeta\right)$ is a pre-quasi closed operator ideal, with $\tau(w)=\sum_{j=0}^{\infty} \psi\left(\left|\Delta_{n+1}^{m}\right| w_{j}||\right), \quad$ for all $w \in \ell_{\psi}\left(\Delta_{n+1}^{m}\right)$ and $\zeta(V)=\tau\left(\left(s_{n}(V)\right)_{n=0}^{\infty}\right)$.
\end{abstract}

Proof. let the conditions be satisfied. Therefore, by using Theorems 3,4 , and 7 , the function $\zeta$ is a pre-quasi norm on the ideal $\left(\ell_{\psi}\left(\Delta_{n+1}^{m}\right)\right)_{\tau}^{\mathrm{S}}$. Assume $V_{j} \in\left(\ell_{\psi}\left(\Delta_{n+1}^{m}\right)\right)_{\tau}^{\mathrm{S}}(W, Z)$ for all $\quad j \in \mathbb{N}$ and $\lim _{j \rightarrow \infty} \zeta\left(V_{j}-V\right)=0$. Since $\mathscr{B}(W, Z) \supseteq\left(\ell_{\psi}\left(\Delta_{n+1}^{m}\right)\right)_{\tau}^{\mathrm{S}}(W, Z)$, we have

$$
\zeta\left(V-V_{j}\right)=\sum_{k=0}^{\infty} \psi\left(\left|\Delta_{n+1}^{m} s_{k}\left(V-V_{j}\right)\right|\right) \geq \psi\left(\left|\Delta_{n+1}^{m} \| V-V_{j}\right|||\right) .
$$

Hence, $\left(V_{j}\right)_{j \in \mathbb{N}}$ is a convergent sequence in $\mathscr{B}(W, Z)$. In addition, $\left(s_{n}\left(V_{j}\right)\right)_{n=0}^{\infty} \in\left(\ell_{\psi}\left(\Delta_{n+1}^{m}\right)\right)_{\tau}$, for each $j \in \mathbb{N}$. From Parts (ii), (iii), and (iv) of Definition 5, we get

$$
\begin{aligned}
\zeta(V) & =\sum_{r=0}^{\infty} \psi\left(\left|\Delta_{n+1}^{m} s_{r}\left(V-V_{j}+V_{j}\right)\right|\right) \leq B\left(\sum_{r=0}^{\infty} \psi\left(\left|\Delta_{n+1}^{m} s\left[\frac{r}{2}\right]\left(V-V_{j}\right)\right|\right)+\sum_{r=0}^{\infty} \psi\left(\left|\Delta_{n+1}^{m} s_{\left[\frac{r}{2}\right]}\left(V_{j}\right)\right|\right)\right) \\
& \leq B \sum_{r=0}^{\infty} \psi\left(\left|\Delta_{n+1}^{m} \| V-V_{j}\right| \mid\right)+B b_{0} \sum_{r=0}^{\infty} \psi\left(\left|\Delta_{n+1}^{m} s_{r}\left(V_{j}\right)\right|\right)<\varepsilon .
\end{aligned}
$$

Therefore, $\left(s_{r}(V)\right)_{r=0}^{\infty} \in\left(\ell_{\psi}\left(\Delta_{n+1}^{m}\right)\right)_{\tau}$. This gives that $V \in\left(\ell_{\psi}\left(\Delta_{n+1}^{m}\right)\right)_{\tau}^{S}(W, Z)$.

Corollary 2. $\left(\left(\ell_{p}\left(\Delta_{n+1}^{m}\right)\right)_{\tau}^{\mathrm{S}}, \zeta\right)$ is a pre-quasi closed and Banach, with $\tau(w)=\sum_{i=0}^{\infty}\left|\Delta_{n+1}^{m}\right| w_{i}||^{p}$, for all $w \in \ell_{p}\left(\Delta_{n+1}^{m}\right)$ and $\zeta(V)=\tau\left(\left(s_{n}(V)\right)_{n=0}^{\infty}\right)$, if $0<p<\infty$ and $\Delta_{n+1}^{m}$ is an absolute nondecreasing.

\section{Small and Simple Pre-Quasi Operator Ideal}

We explain the sufficient conditions on $\ell_{\psi}\left(\Delta_{n+1}^{m}\right)$ such that the strictly inclusion relation of $\left(\ell_{\psi}\left(\Delta_{n+1}^{m}\right)\right)^{\mathrm{S}}$, for different $\psi$ and $\Delta_{n+1}^{m}$, has been happened.

Theorem 11. For any infinite dimensional Banach spaces $W$ and $Z$. Let $\psi_{1}$ and $\psi_{2}$ be two Orlicz functions satisfying $\delta_{2}$-condition with $\psi_{2}(t)<\psi_{1}(t)$ for all $t \in(0, \infty)$ and $\Delta_{n}^{m}$ is an absolute nondecreasing, for all $n, m \in \mathbb{N}$, then

$$
\left(\ell_{r_{2}}\left(\Delta_{n+1}^{m}\right)\right)^{S}(W, Z) \underset{\ddagger}{\subset}\left(\ell_{j_{2}}\left(\Delta_{n+1}^{m+1}\right)\right)^{S}(W, Z) \underset{\ddagger}{\subset} \mathscr{B}(W, Z) .
$$

Proof. Let the conditions be satisfied. If $V \in\left(\ell_{\psi_{1}}\left(\Delta_{n+2}^{m}\right)\right)^{\mathrm{S}}(W, Z)$, we have $\left(s_{i}(V)\right) \in \ell_{\psi_{1}}\left(\Delta_{n+2}^{m}\right)$. One can see

$$
\sum_{j=0}^{\infty} \psi_{2}\left(\left|\Delta_{n+1}^{m+1} s_{j}(V)\right|\right)<\sum_{j=0}^{\infty} \psi_{1}\left(\left|\Delta_{n+2}^{m} s_{j}(V)\right|\right)<\infty .
$$

Therefore, $V \in\left(\ell_{\psi_{2}}\left(\Delta_{n+1}^{m+1}\right)\right)^{\mathrm{S}}(W, Z)$. Next, if we choose $\left(s_{j}(V)\right)_{j=0}^{\infty}$ such that $\psi_{1}\left(\left|\Delta_{n+2}^{m} s_{j}(V)\right|\right)=(j+1)^{-1}$ and $\psi_{2}\left(\left|\Delta_{n+2}^{m} s_{j}(V)\right|\right)=(j+1)^{-2}$, for $n, m \in \mathbb{N}$, we can find $V \in \mathscr{B}(W, Z)$ with $\sum_{j=0}^{\infty} \psi_{1}\left(\left|\Delta_{n+2}^{m} s_{j}(V)\right|\right)=\sum_{j=0}^{\infty}(j+1)^{-1}=$ $\infty \quad$ and $\quad \sum_{j=0}^{\infty} \psi_{2}\left(\left(\left|\Delta_{n+2}^{m} s_{j}(V)\right|\right)\right)=\sum_{j=0}^{\infty}(j+1)^{-2}<\infty$. Therefore, $\quad \ell_{\psi_{2}}\left(\Delta_{n+2}^{m}\right) \subseteq \ell_{\psi_{2}}\left(\Delta_{n+1}^{m+1}\right)$. Therefore, $V \notin\left(\ell_{\psi_{1}}\left(\Delta_{n+2}^{m}\right)\right)^{\mathrm{S}}(W, Z)$ and $V \in\left(\ell_{\psi_{2}}\left(\Delta_{n+1}^{m+1}\right)\right)^{\mathrm{S}}(W, Z)$. Clearly, $\quad\left(\ell_{\psi_{2}}\left(\Delta_{n+1}^{m+1}\right)\right)^{\mathrm{s}}(W, Z) \subset \mathscr{B}(W, Z)$. Choose $\left(s_{j}(V)\right)_{j=0}^{\infty}$ such that $\Delta_{n+1}^{m+1} s_{j}(V)=(j+1)^{-1}$, for $n, m \in \mathbb{N}$. We have $V \in \mathscr{B}(W, Z)$ such that $V \notin\left(\ell_{\psi_{2}}\left(\Delta_{n+1}^{m+1}\right)\right)^{\mathrm{s}}(W, Z)$.

Corollary 3. For any infinite dimensional Banach spaces $W$ and $Z, j \geq r>0$ and absolute nondecreasing $\Delta_{n}^{m}$, for every $n, m \in \mathbb{N}$, then 


$$
\left(\ell_{\psi_{2}}\left(\Delta_{n+1}^{m}\right)\right)^{\mathrm{S}}(W, Z) \underset{\ddagger}{\subset}\left(\ell_{j}\left(\Delta_{n+1}^{m+1}\right)\right)^{\mathrm{S}}(W, Z) \underset{\ddagger}{\subset} \mathscr{B}(W, Z) .
$$

We study the conditions such that the class $\left(\ell_{\psi}\left(\Delta_{n+1}^{m}\right)\right)^{a p p}$ is small.

Theorem 12. For any Banach spaces $W$ and $Z$ with $\operatorname{dim}(W)=\operatorname{dim}(Z)=\infty$. Let $\psi$ be an Orlicz function satisfying $\delta_{2}$-condition and $\Delta_{n+1}^{m}$ be an absolute nondecreasing, then the class $\left(\ell_{\psi}\left(\Delta_{n+1}^{m}\right)\right)^{a p p}$ is small.
Proof. Let the conditions be verified. Therefore, $\left(\left(\ell_{\psi}\left(\Delta_{n+1}^{m}\right)\right)^{\text {app }}, \zeta\right)$ be a pre-quasi Banach operator ideal, where $\zeta(V)=\sum_{k=0}^{\infty} \psi\left(\left|\Delta_{n+1}^{m} \alpha_{k}(V)\right|\right)$.

Let $\left(\ell_{\psi}\left(\Delta_{n+1}^{m}\right)\right)^{\mathrm{app}}(W, Z)=\mathscr{B}(W, Z)$, so there is $\delta>0$ with $\zeta(V) \leq \delta\|V\|$ for all $V \in \mathscr{B}(W, Z)$. From Dvoretzky's theorem [11] for $j \in \mathbb{N}$, there are subspaces $M_{j}$ and quotient spaces $W / N_{j}$ of $Z$. By isomorphisms $A_{j}$ and $H_{j}$ will be mapped $Z$ onto $\ell_{2}^{j}$ with $\left\|H_{j}\right\|\left\|H_{j}^{-1}\right\| \leq 2$ and $\left\|A_{j}\right\|\left\|A_{j}^{-1}\right\| \leq 2$. Let $J_{j}$ be the natural embedding map from $M_{j}$ into $Z$ and $Q_{j}$ be the quotient map from $W$ onto $W / N_{j}$. If we denote the Bernstein numbers [11] by $u_{j}$, we have

$$
\begin{aligned}
1 & =u_{k}\left(I_{j}\right)=u_{k}\left(A_{j} A_{j}^{-1} I_{j} H_{j} H_{j}^{-1}\right) \leq\left\|A_{j}\right\| u_{k}\left(A_{j}^{-1} I_{j} H_{j}\right)\left\|H_{j}^{-1}\right\|=\left\|A_{j}\right\| u_{k}\left(J_{j} A_{j}^{-1} I_{j} H_{j}\right)\left\|H_{j}^{-1}\right\| \\
& \leq\left\|A_{j}\right\| d_{k}\left(J_{j} A_{j}^{-1} I_{j} H_{j}\right)\left\|H_{j}^{-1}\right\|=\left\|A_{j}\right\| d_{k}\left(J_{j} A_{j}^{-1} I_{j} H_{j} Q_{j}\right)\left\|H_{j}^{-1}\right\| \leq\left\|A_{j}\right\| \alpha_{k}\left(J_{j} A_{j}^{-1} I_{j} H_{j} Q_{j}\right)\left\|H_{j}^{-1}\right\|,
\end{aligned}
$$

for $0 \leq k \leq i$. Since $\psi$ is an Orlicz function satisfying $\delta_{2}$-condition, we have

$$
\begin{aligned}
& (i+1) \psi(1) \leq a\left\|A_{j}\right\|\left\|H_{j}^{-1}\right\| \sum_{k=0}^{i} \psi\left(\left|\Delta_{n+1}^{m} \alpha_{k}\left(J_{j} A_{j}^{-1} I_{j} H_{j} Q_{j}\right)\right|\right) \Rightarrow \\
& (i+1) \psi(1) \leq a\left\|A_{j}\right\|\left\|H_{j}^{-1}\right\| g\left(J_{j} A_{j}^{-1} I_{j} H_{j} Q_{j}\right) \Rightarrow(i+1) \psi(1) \leq a \delta\left\|A_{j}\right\|\left\|H_{j}^{-1}\right\|\left\|J_{j} A_{j}^{-1} I_{j} H_{j} Q_{j}\right\| \Rightarrow \\
& (i+1) \psi(1) \leq a \delta\left\|A_{j}\right\|\left\|H_{j}^{-1}\right\|\left\|J_{j} A_{j}^{-1}\right\|\left\|I_{j}\right\|\left\|H_{j} Q_{j}\right\|=L \delta\left\|A_{j}\right\|\left\|H_{j}^{-1}\right\|\left\|A_{j}^{-1}\right\|\left\|I_{j}\right\|\left\|H_{j}\right\| \Rightarrow \\
& (i+1) \psi(1) \leq 4 a \delta,
\end{aligned}
$$

for some $a \geq 1$. Since $i$ is an arbitrary, we have a contradiction. So, $W$ and $Z$ cannot be infinite dimensional while $\left(\ell_{\psi}\left(\Delta_{n+1}^{m}\right)\right)^{\mathrm{app}}(W, Z)=\mathscr{B}(W, Z)$.

By the same manner, one can prove that the class $\left(\ell_{\psi}\left(\Delta_{n+1}^{m}\right)\right)^{\mathrm{Kol}}$ is small.

Theorem 13. Hold any Banach spaces $W$ and $Z$ with $\operatorname{dim}(W)=\operatorname{dim}(Z)=\infty$. Let $v$ be an Orlicz function satisfying $\delta_{2}$-condition and $\Delta_{n+1}^{m}$ is an absolute nondecreasing, then the class $\left(\ell_{\psi}\left(\Delta_{n+1}^{m}\right)\right)^{K o} l^{n+1}$ is small.

For which $\ell_{\psi}\left(\Delta_{n+1}^{m}\right)$, is $\left(\ell_{\psi}\left(\Delta_{n+1}^{m}\right)\right)^{\mathrm{S}}$ simple?

Theorem 14. For any infinite dimensional Banach spaces $W$ and Z. Let $\psi_{1}$ and $\psi_{2}$ be two Orlicz functions satisfying $\delta_{2}$-condition with $\psi_{2}(t)<\psi_{1}(t)$ for all $t \in(0, \infty)$ and $\Delta_{n}^{m}$ is an absolute nondecreasing, for all $n, m \in \mathbb{N}$, then

$$
\mathscr{B}\left(\left(\ell_{\psi_{2}}\left(\Delta_{n+1}^{m+1}\right)\right)^{S},\left(\ell_{\psi_{2}}\left(\Delta_{n+1}^{m}\right)\right)^{S}\right)=\Upsilon\left(\left(\ell_{\psi_{2}}\left(\Delta_{n+1}^{m+1}\right)\right)^{S},\left(\ell_{\psi_{2}}\left(\Delta_{n+1}^{m}\right)\right)^{\mathrm{S}}\right) .
$$

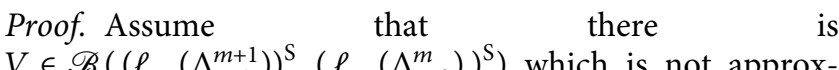
$V \in \mathscr{B}\left(\left(\ell_{\psi_{2}}\left(\Delta_{n+1}^{m+1}\right)\right)^{\mathrm{S}},\left(\ell_{\psi_{1}}\left(\Delta_{n+2}^{m}\right)\right)^{\mathrm{S}}\right)$ which is not approximable. By Lemma 1 , we have $G \in \mathscr{B}\left(\left(\ell_{\psi_{2}}\left(\Delta_{n+1}^{m+1}\right)\right)^{S}\right)$ and

$B \in \mathscr{B}\left(\left(\ell_{\psi_{1}}\left(\Delta_{n+2}^{m}\right)\right)^{S}\right)$ with $B V G I_{k}=I_{k}$. Therefore, for all $k \in \mathbb{N}$, we get

$$
\left\|I_{k}\right\|_{\left(\ell_{\psi_{1}}\left(\Delta_{n+2}^{m}\right)\right)^{s}}=\sum_{n=0}^{\infty} \psi_{1}\left(\left|\Delta_{n+2}^{m} s_{n}\left(I_{k}\right)\right|\right) \leq\|B V G\|\left\|I_{k}\right\|_{\left(\ell_{\psi_{2}}\left(\Delta_{n+1}^{m+1}\right)\right)^{s}} \leq \sum_{n=0}^{\infty} \psi_{2}\left(\left|\Delta_{n+1}^{m+1} s_{n}\left(I_{k}\right)\right|\right) .
$$

From Theorem 11, we obtain a contradiction. Hence, $V \in \Upsilon\left(\left(\ell_{\psi_{2}}\left(\Delta_{n+1}^{m+1}\right)\right)^{\mathrm{S}},\left(\ell_{\psi_{1}}\left(\Delta_{n+2}^{m}\right)\right)^{\mathrm{S}}\right)$.
Corollary 4. For any infinite dimensional Banach spaces $W$ and Z. Let $\psi_{1}$ and $\psi_{2}$ be two Orlicz functions satisfying 
$\delta_{2}$-condition with $\psi_{2}(t)<\psi_{1}(t)$ for all $t \in(0, \infty)$ and $\Delta_{n}^{m}$ is an absolute nondecreasing, for all $n, m \in \mathbb{N}$, then

$$
\mathscr{B}\left(\left(\ell_{\psi_{2}}\left(\Delta_{n+1}^{m+1}\right)\right)^{\mathrm{S}},\left(\ell_{\psi_{2}}\left(\Delta_{n+1}^{m}\right)\right)^{\mathrm{S}}\right)=\mathscr{B}_{c}\left(\left(\ell_{\psi_{2}}\left(\Delta_{n+1}^{m+1}\right)\right)^{\mathrm{S}},\left(\ell_{\psi_{2}}\left(\Delta_{n+1}^{m}\right)\right)^{\mathrm{S}}\right) .
$$

Proof. Clearly, since each approximable operator is compact.

Theorem 15. Pick up any Banach spaces $W$ and $Z$ with $\operatorname{dim}(W)=\operatorname{dim}(Z)=\infty$. If $\psi$ is an Orlicz function satisfying $\delta_{2}$-condition and $\Delta_{n}^{m}$ is an absolute nondecreasing, then the class $\left(\ell_{\psi}\left(\Delta_{n+1}^{m}\right)\right)_{\tau}^{S}$ is simple.

Proof. Suppose that there is $V \in \mathscr{B}_{c}\left(\left(\ell_{\psi}\left(\Delta_{n+1}^{m}\right)\right)_{\tau}^{\mathrm{S}}\right)$ and $V \notin \Upsilon\left(\left(\ell_{\psi}\left(\Delta_{n+1}^{m}\right)\right)_{\tau}^{S}\right)$. Therefore, from Lemma 1, one can find that $A, B \in \mathscr{B}\left(\left(\ell_{\psi}^{\tau}\left(\Delta_{n+1}^{m}\right)\right)_{\tau}^{\mathrm{S}}\right)$ with $B V A I_{k}=I_{k}$. This means

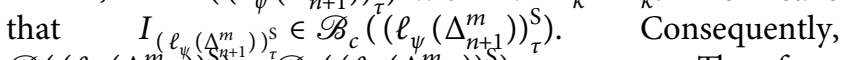
$\mathscr{B}\left(\left(\ell_{\psi}\left(\Delta_{n+1}^{m}\right)\right)_{\tau}^{s+1}\right)={ }_{\tau}^{s} \mathscr{B}_{c}\left(\left(\ell_{\psi}\left(\Delta_{n+1}^{m}\right)\right)_{\tau}^{S}\right)$. Therefore, $\mathscr{B}\left(\left(\ell_{\psi}\left(\Delta_{n+1}^{m}\right)\right)_{\tau}^{\bar{S}}\right)$ includes one and only one nontrivial closed ideal $\Upsilon\left(\left(\ell_{\psi}\left(\Delta_{n+1}^{m}\right)\right)_{\tau}^{\mathrm{S}}\right)$.

\section{Eigenvalues of $s$-Type Orlicz Generalized Difference Sequence Space}

We explain here the sufficient conditions on $\ell_{\psi}\left(\Delta_{n+1}^{m}\right)$ such that $\left(\ell_{\psi}\left(\Delta_{n+1}^{m}\right)\right)^{\mathrm{S}}$ equals $\left(\ell_{\psi}\left(\Delta_{n+1}^{m}\right)\right)^{\nu}$.

Theorem 16. Pick up any Banach spaces $W$ and $Z$ with $\operatorname{dim}(W)=\operatorname{dim}(Z)=\infty$. If $\psi$ is an Orlicz function satisfying $\delta_{2}$-condition and $\Delta_{n+1}^{m}$ is an absolute nondecreasing, then

$$
\left(\ell_{\psi}\left(\Delta_{n+1}^{m}\right)\right)^{\mathrm{S}}(W, Z)=\left(\ell_{\psi}\left(\Delta_{n+1}^{m}\right)\right)^{\nu}(W, Z) .
$$

Proof. Suppose $V \in\left(\ell_{\psi}\left(\Delta_{n+1}^{m}\right)\right)^{S}(W, Z)$ and then $\left(s_{r}(V)\right)_{r=0}^{\infty} \in \ell_{\psi}\left(\Delta_{n+1}^{m}\right)$, we have $\sum_{r=0}^{\infty} \psi\left(\left(\left|\Delta_{n+1}^{m} s_{r}(V)\right|\right)\right)<\infty$. Since $\Delta_{n+1}^{m}$ is continuous, so $\lim _{r \rightarrow \infty} s_{r}(V)=0$. Let $\| V-$ $s_{r}(V) I \|$ be an invertible, for all $r \in \mathbb{N}$, then $\left\|V-s_{r}(V) I\right\|^{-1}$ exists and bounded, for each $r \in \mathbb{N}$. Therefore, $\lim _{r \rightarrow \infty}\left\|V-s_{r}(V) I\right\|^{-1}=\|V\|^{-1} \quad$ with $\quad V^{-1} \in \mathscr{B}(Z, W)$. From the pre-quasi operator ideal of $\left(\left(\ell_{\psi}\left(\Delta_{n+1}^{m}\right)\right)^{S}, \zeta\right)$, one has

$$
\begin{aligned}
I & =V V^{-1} \in\left(\ell_{\psi}\left(\Delta_{n+1}^{m}\right)\right)^{\mathrm{S}}(Z) \Longrightarrow\left(s_{r}(I)\right)_{r=0}^{\infty} \in \ell_{\psi}\left(\Delta_{n+1}^{m}\right) \\
& \Rightarrow \lim _{r \longrightarrow \infty} s_{r}(I)=0 .
\end{aligned}
$$

Therefore, $\lim _{r \rightarrow \infty} s_{r}(I)=1$. We have a contradiction, and then $\left\|V-s_{r}(V) I\right\|$ is not invertible, for all $r \in \mathbb{N}$. Hence, $\left(s_{r}(V)\right)_{r=0}^{\infty}$ is the eigenvalues of $V$. Conversely, if $V \in\left(\ell_{\psi}\left(\Delta_{n+1}^{m}\right)\right)^{\nu}(W, Z)$, then $\left(\nu_{r}(V)\right)_{r=0}^{\infty} \in \ell_{\psi}\left(\Delta_{n+1}^{m}\right)$ and $\left\|V-v_{r}(V) I\right\|=0$, for all $n \in \mathbb{N}$. This gives that $V=v_{r}(V) I$, for all $r \in \mathbb{N}$, and then $s_{r}(V)=s_{r}\left(v_{r}(V) I\right)=\left|v_{r}(V)\right|$, for all $r \in \mathbb{N}$. Therefore, $\quad\left(s_{r}(V)\right)_{r=0}^{\infty} \in \ell_{\psi}\left(\Delta_{n+1}^{m}\right), \quad$ so $V \in\left(\ell_{\psi}\left(\Delta_{n+1}^{m}\right)\right)^{\mathrm{S}}(W, Z)$. This completes the proof.

\section{Conclusion}

We have introduced the concept of the pre-quasi norm on the new sequence space generated by the domain of generalized backward difference operator in Orlicz sequence space. This space is not operator ideal since it is not solid. However, if the generalized backward difference operator is an absolute nondecreasing and Orlicz function satisfies $\delta_{2}$-condition, then the operator ideal constructed by this sequence space and $s$-numbers will be Banach, closed, small, and simple. Finally, we have found the spectrum of all operators contained in this operator ideal.

\section{Data Availability}

The data used to support the findings of the study are available from the corresponding author upon request.

\section{Ethical Approval}

This article does not contain any studies with human participants or animals performed by any of the authors.

\section{Conflicts of Interest}

The authors declare that they have no conflicts of interest.

\section{Authors' Contributions}

All the authors contributed equally to the writing of this paper and read and approved the final manuscript.

\section{Acknowledgments}

This work was funded by the University of Jeddah, Saudi Arabia (under grant no. UJ-02-054-DR). The authors, therefore, acknowledge with thanks the university technical and financial support.

\section{References}

[1] B. C. Tripathy, A. Esi, and B. K. Tripathy, "On a new type of generalized difference Cesáro sequence spaces," Soochow Journal of Mathematics, vol. 31, no. 3, pp. 333-340, 2005.

[2] M. Et and R. Çolak, "On some generalized difference spaces," Soochow Journal of Mathematics, vol. 21, pp. 377-386, 1995.

[3] B. C. Tripathy and A. Esi, "A new type of sequence spaces," International Journal of Science \& Technology, vol. 1, no. 1, pp. 11-14, 2006.

[4] H. Kizmaz, "On certain sequence spaces," Canadian Mathematical Bulletin, vol. 24, no. 2, pp. 169-176, 1981. 
[5] A. A. Bakery and A. R. Abou Elmatty, "A note on nakano generalized difference sequence space," Advances in Difference Equations, vol. 2020620 pages, 2020.

[6] M. A. Krasnoselskii and Y. B. Rutickii, Convex Functions and Orlicz Spaces, Cambridge University Press, Gorningen, Netherlands, 1961.

[7] W. Orlicz and Ü. Raume, $\left(L^{M}\right)$ Bulletin of the Polish Academy of Sciences A, pp. 93-107, 1936.

[8] J. Lindenstrauss and L. Tzafriri, "On orlicz sequence spaces," Israel Journal of Mathematics, vol. 10, no. 3, pp. 379-390, 1971.

[9] S. A. Mohiuddine, K. Raj, M. Mursaleen, and A. Alotaibi, "Linear isomorphic spaces of fractional-order difference operators," Alexandria Engineering Journal, vol. 60, no. 1, 2020.

[10] T. Yaying, B. Hazarika, S. A. Mohiuddine, M. Mursaleen, and K. J. Ansari, "Sequence spaces derived by the triple band generalized fibonacci difference operator," Advances in Difference Equations, vol. 2020639 pages, 2020.

[11] A. Pietsch, Operator Ideals, North-Holland Publishing Company, Amsterdam, Netherlands, 1980.

[12] A. Pietsch, "Small ideals of operators," Studia Mathematica, vol. 51, no. 3, pp. 265-267, 1974.

[13] B. M. Makarov and N. Faried, Some Properties of Operator Ideals Constructed by s Numbers, Theory of Operators in Functional Spaces, Academy of Science, Siberian section, Novosibirsk, Russia, in Russian, 1977.

[14] N. Faried and A. A. Bakery, "Small operator ideals formed by s numbers on generalized Cesáro and orlicz sequence spaces," Journal of Inequalities and Applications, vol. 2018357 pages, 2018.

[15] E. E. Kara and M. Başarir, "On compact operators and some euler $(B(m)$-difference sequence spaces," Journal of Mathematical Analysis and Applications, vol. 379, no. 2, pp. 499-511, 2011.

[16] M. İlkhan, S. Demiriz, and E. E. Kara, "Multiplication operators on Cesáro second order function spaces," Positivity, vol. 24, pp. 605-614, 2020.

[17] B. Altay and F. Başar, "Generalization of the sequence space $\ell(\mathrm{P})$ derived by weighted means," Journal of Mathematical Analysis and Applications, vol. 330, no. 1, pp. 147-185, 2007. 